\title{
High Resolution SAR Interferometry: influence of local topography in the context of glacier monitoring
}

\author{
G. Vasile*ף, I. Petillot*, A. Julea*ף\|, E. Trouvé*, Ph. Bolon*, \\ L. Bombrun ${ }^{\dagger}$, M. Gay ${ }^{\dagger}$, T. Landes ${ }^{\ddagger}$, P. Grussenmeyer ${ }^{\ddagger}$, and J.-M. Nicolas ${ }^{\S}$
}

* Université de Savoie, ESIA/LISTIC - BP 806 - F-74016 Annecy Cedex, FRANCE

Tel: +33 450096548 - Email: \{g.vasile|ivan.petillot|andreea.julea|trouve|bolon\}@univ-savoie.fr

${ }^{\dagger}$ Institut National Polytechnique de Grenoble/LIS - BP 46 - 38402 Saint-Martin-d'Hères, FRANCE

Tel: +33476826256 - Email: \{lionel.bombrun|michel.gay\}@ lis.inpg.fr

$\ddagger$ INSA Strasbourg - Equipe Photogrammétrie et Géomatique - 24, Bd de la Victoire, 67084 Strasbourg Cedex, FRANCE

Tel: +33 388144733 - Email: \{tania.landes|pierre.grussenmeyer\}@insa-strasbourg.fr

$\S$ Ecole Nationale Supérieure des Télécommunications, LTCI - 46, Rue Barrault - 75013 Paris, FRANCE

Tel: +33145818129 - Email: nicolas@tsi.enst.fr

I Universitatea Politehnica Bucuresti, LAPI - Bucharest, ROMANIA - Tel: +4021 4024683

|| Institutul de Stiinte Spatiale - Bucuresti, ROMANIA - Tel/fax +4021 4574471

\begin{abstract}
SAR interferometric data offers the opportunity to measure temperate glacier surface topography and displacement between the two acquisitions. Recently, reliable estimates of the phase gradient given by interferogram local frequencies become mandatory with the increase of the SAR resolution. In this paper, an original 2 -step method for estimating local frequencies is proposed. The $2 \mathrm{D}$ phase signal is considered to have two deterministic components corresponding to low-resolution fringes and high-resolution patterns due for instance to the micro-relief. The first step of the proposed algorithm consists in the low-resolution phase flattening. In the second step the local high-resolution frequencies are estimated from the phase auto-correlation functions computed on adaptive neighborhoods using only the pixels which belong to the same HR spatial feature and respect the "local stationarity" hypothesis. Results with both real ERS 1/2 tandem and simulated TerraSAR-X interferograms are presented to illustrate the potential of the proposed method.
\end{abstract}

\section{INTRODUCTION}

With an increased resolution, the new space-borne SAR images should be an important source of information for glacier activity monitoring, by providing regular measurements such as surface topography, velocity fields or rocks and crevasses cartography. After successful SAR experiments on Arctic and polar region glaciers [1], the study of the alpine temperate glaciers proved to be more complex. Some experiments carried out with existing space-borne SAR interferometric (InSAR) data have shown that only specific data such as ERS 1/2 tandem couples provide information on well-oriented glaciers at certain time of the year [2]. But the characteristics of the future space-borne SAR missions such as TerraSAR-X (14-day repeat cycle, left/right looking, various incidence angles, up to 2-meter resolution, dual polarisation...) reinforce the potential of interferometric measurements over alpine glaciers.

The existing standard interferometric processors (the Differential Interferometric Automated Process Applied to Survey Of Nature - DIAPASON or the Repeated Orbit Interferometry Package - ROI-PAC) provide a complete
D-InSAR processing chain, which was already tested and successfully applied in the context of SAR interferometry to measure accurate displacements (volcanology or seismology). Although these processors are built from standard well-established InSAR algorithms as co-registration of interferograms, phase scaling and unwrapping, this procedure is often error-prone when applied to temperate glacier monitoring because of the complex glacier topography or the phase noise and discontinuities at glacier boundaries.

With the resolution of the data which have been available up to now (about $20 \mathrm{~m}$ with ERS images), the amplitude is often difficult to use to extract precise information on specific features. The next generation of SAR satellites should provide meter resolution images and fully polarimetric data, which will be very useful to detect different features and backscattering mechanisms and to improve the extraction of the geometric deterministic component of the interferometric phase by adaptive techniques [3]. The two main limitations of the glacier InSAR measurements are the temporal decorrelation and the need to separate the topographical fringes from the displacement fringes. Among the different techniques which can be used, the safest one consists in using a Digital Terrain Model (DTM) of the target area. The DTM accuracy requirement increases with the baseline between the two SAR acquisitions, which makes interferograms more and more sensitive to the topography, especially in the high relief areas of Alpine glaciers.

In this context, reliable estimates of the phase gradient within the interferogram become more and more necessary with the increase of the SAR resolution: smaller ground resolution cells will reveal the local topographic variations (micro-relief) and larger baselines (smaller altitude of ambiguity) will transform them into local fringe pattern difficult to detect without using adaptive techniques. A previous approach based on the estimation 
of local frequencies proved to be rather efficient with low resolution data. If correctly estimated, local frequencies can be used in the InSAR processing at different stages such as interferogram filtering or phase unwrapping [4]. In this paper, we propose to improve this approach and to adapt it to the new context of high resolution interferograms. An original 2-step method for estimating local frequencies is presented. Results using two different data sets are presented over the Mer-de-Glace and Leschaux glaciers which are located in the Mont-Blanc region: a real ERS tandem interferogram containing topographic and displacement fringes and a simulated high resolution (HR) interferogram obtained from a HR DTM computed from aerial photographs.

\section{HIGH FREQUENCY FRINGE PATTERN ANALYSIS}

\section{A. Proposed model}

In each pixel of the interferogram, the $2 \mathrm{D}$ phase signal $\phi$ is considered to have two deterministic components, namely the low-resolution phase $\phi_{L R}$ which corresponds to low-resolution items (fields, mountains...) and the highresolution phase $\phi_{H R}$ which appears in HR data (small local topography variations as ice-cracks or larger rocks), and one random component corresponding to the phase noise $\phi_{n}$. Accordingly, the analytical phase signal can be written as:

$$
e^{i \phi}=e^{i \phi_{L R}} e^{i \phi_{H} R} e^{i \phi_{n}}
$$

The first step of the proposed algorithm consists in estimating the low-resolution phase and removing the associated fringe pattern. After reducing the initial resolution of the interferogram, a modified version of the MUSIC algorithm [5] is applied for estimating the 2D local frequency $\left(f_{x}^{L R}, f_{y}^{L R}\right)$ of a fringe pattern within a squared fixed-size centered neighborhood. This lowresolution frequency is used in each pixel $(m, n)$ of the full resolution interferogram to approximatively flatten the global fringe pattern in surrounding pixels $(k, l)$ :

$$
\phi_{f l}(k, l)=\phi(k, l)-2 \pi\left((k-m) f_{x}^{L R}+(l-n) f_{y}^{L R}\right) .
$$

The resulting phase signal $e^{\phi_{f l}}$ exhibits the local differences between the $2 \mathrm{D}$ sine-wave model and the real HR fringe pattern.

\section{B. Spatial support}

In the second step, the local high-resolution frequencies are estimated. The proposed procedure consists in gathering only the pixels which belong to the same HR spatial feature and respect the "local stationarity" hypothesis [6]. Under the assumption that small homogeneous regions within the two intensity images correspond to ground areas with an homogeneous cover which should respect the stationarity hypothesis, the multi-resolution technique employed in this paper is the Amplitude-Driven-AdaptiveNeighborhood (ADAN) [3].

\section{Auto-correlation functions}

Using as spatial support the obtained adaptive neighborhood, the two 1D autocorrelation functions of the LRflattened phase signal given by Eq. 2 are computed. Using
$1 \mathrm{D}$ notation and a phase model $\phi(z)=z f_{z}+\phi_{n}$, the autocorrelation coefficients are defined by:

$$
\gamma(p)=\left\langle e^{\jmath\left(\phi(z+p)+\phi_{n}(z+p)\right)} \cdot e^{-\jmath\left(\phi(z)+\phi_{n}(z)\right)}\right\rangle
$$

Under the assumption that the phase noise is an independent and identically distributed (iid) random process, we obtain:

$$
\gamma(p)=K e^{\jmath 2 \pi p f_{z}}
$$

where $K=\left|\left\langle e^{\jmath \phi_{n}(0)}\right\rangle\right|^{2}$ is a real coefficient which does not affect the autocorrelation phase anymore. Accordingly, our algorithm estimates the frequency as:

$$
\widehat{f}_{z}=\arg \left[\sum_{p} \frac{N(p)+N(p+1)}{2} \gamma(p+1) \cdot \overline{\gamma(p)}\right]
$$

where $N(p)$ is the number of pairs of pixels belonging to to the adaptive neighborhood that can be used to estimate $\gamma(p)$.

The proposed algorithm applied in each direction allows to obtain the expected values $\left(f_{x}^{H R}, f_{y}^{H R}\right)$ measuring the high resolution component of the fringe pattern local frequency.

\section{RESULTS AND DISCUSSION}

The proposed method has been tested on the Chamonix Mont-Blanc test site which is located in the Alps, near the borders between France, Italy and Switzerland $\left(45^{\circ} 50^{\prime} \mathrm{N}\right.$, $\left.6^{\circ} 51^{\prime} \mathrm{E}\right)$. It includes the Aiguille-Verte (4122 m ASL), the Chamonix valley (1000 $\mathrm{m}$ ASL) and two well-known instrumented glaciers: Mer-de-Glace and Leschaux.

\section{A. Real ERS 1/2 TANDEM InSAR data}

In order to test the feasibility of the proposed method on real data, an ERS 1/2 tandem pair has been selected (Tab. III-A) and processed by the ROI-PAC software.

\section{TABLE I}

ERS SAR RAW DATA ACQUIRED OVER THE TEST AREA.

\begin{tabular}{||c||c|c|c|c|c|c||}
\hline \hline Date & Satel. & Orbit & Frame & Pass & $B_{\perp}$ & $e_{a}$ \\
\hline 95.12 .31 & ERS-1 & 23328 & 2673 & Des. & $208 \mathrm{~m}$ & $45 \mathrm{~m}$ \\
96.01 .01 & ERS-2 & 03655 & & & & \\
\hline \hline
\end{tabular}

Starting from the RAW data, SLC images are synthesized and corregistered and a $1 \times 5$ complex multilooking with orbital fringe correction is applied to obtain the amplitude and phase images illustrated in Figs. 1-a,-b. The resulting images have approximately 20-meter ground resolution squared pixels. The fringe pattern which can be observed in the Fig. 1-a has a relatively high frequency due to the small altitude of ambiguity $e_{a}$. Moreover, along the two glaciers the topographic fringes are superposed on the glacier displacement phase component.

The results of the 2D local frequency estimation performed at low resolution (LR), at high resolution (HR) after LR-flattening, and the final combined estimates are illustrated in Fig. 2 by the orientation of the corresponding phase gradient. The LR result is as expected smooth and robust but it does not follow the local variations of the fringe pattern. This information is obtained in the HR 
a)

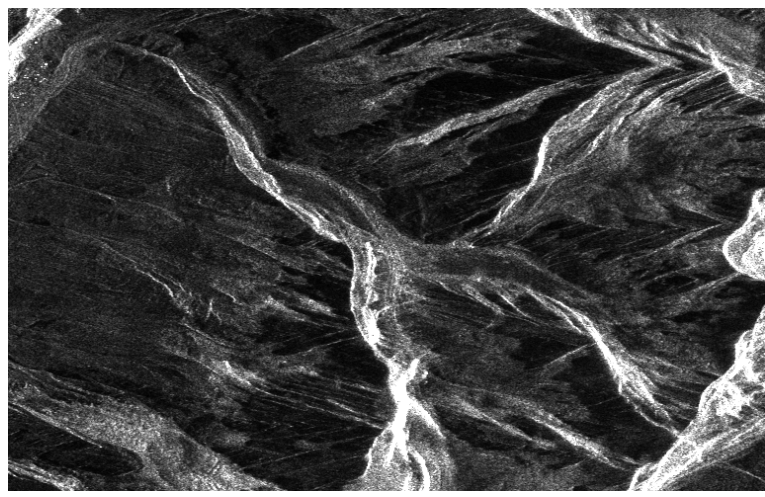

b)
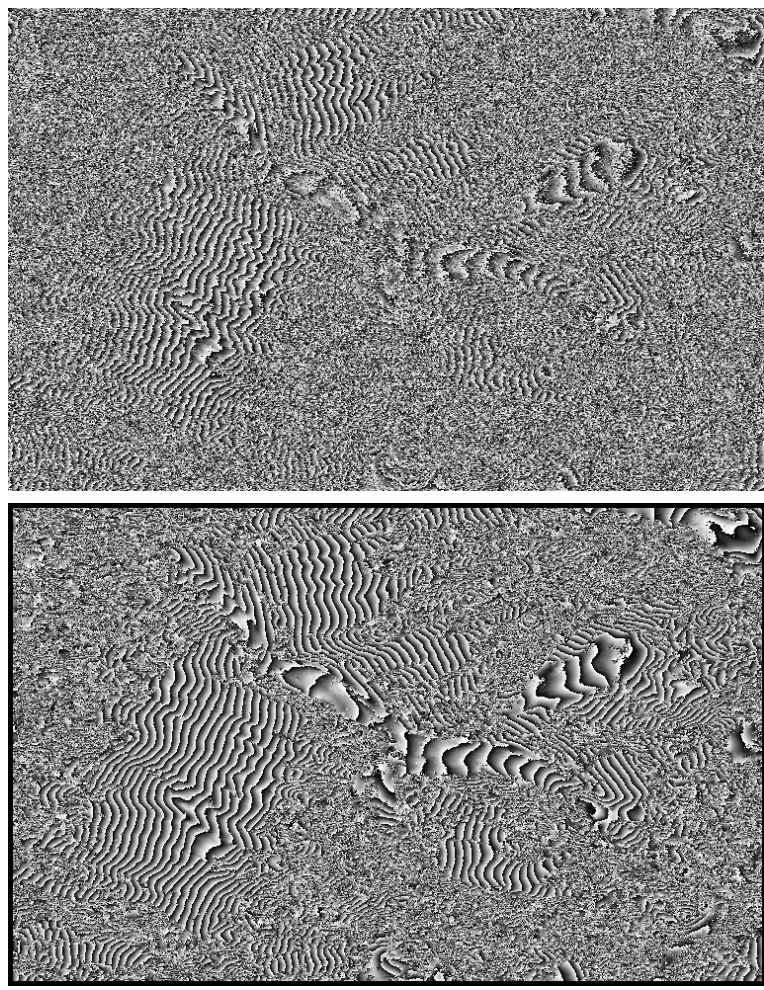

Fig. 1. TANDEM ERS 1/2 interferogram: a) SAR amplitude, b) original phase, c) ADAN filtered phase.

result and the sum of these two estimates provides a robust and precise estimate of the local fringe pattern.

To illustrate the interest and the accuracy of this result, the original noisy interferogram was filtered with the Amplitude-Driven-Adaptive-Neighborhood (ADAN) filter [3], using the deterministic fringe pattern compensation provided by the HR and LR local frequencies estimation (Fig. 1-c). The filtered interferogram preserves well the stationarity in the foldover polluted areas. Moreover, the glacier is not connected with the surrounding terrain and still remains a disconnected phase pattern (useful for eventual D-InSAR phase unwrapping).

\section{B. Simulated TERRASAR-X InSAR data}

In order to illustrate the proposed method on HR data over Alpine glaciers, a 2-meter resolution DTM of the Mer-de-Glace [2] has been used to simulate the SAR intensity of TerraSAR-X images using the method proposed in [7]. The parameters used in the simulation are presented in Tab. III-B. Moreover, the altitude information a)

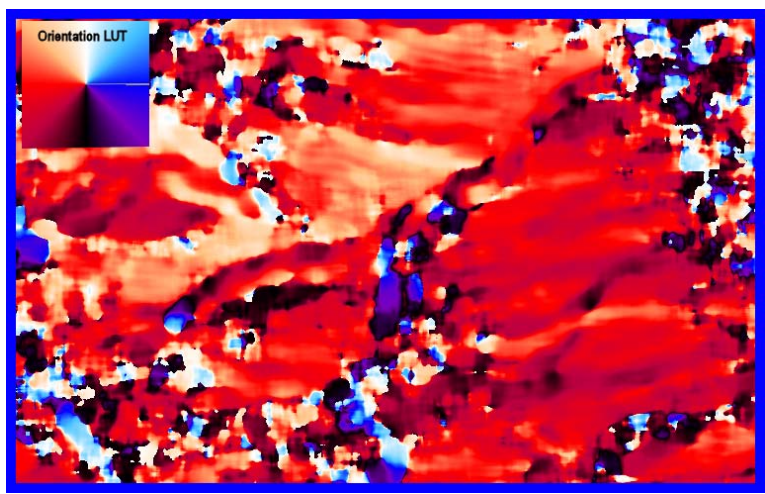

b)

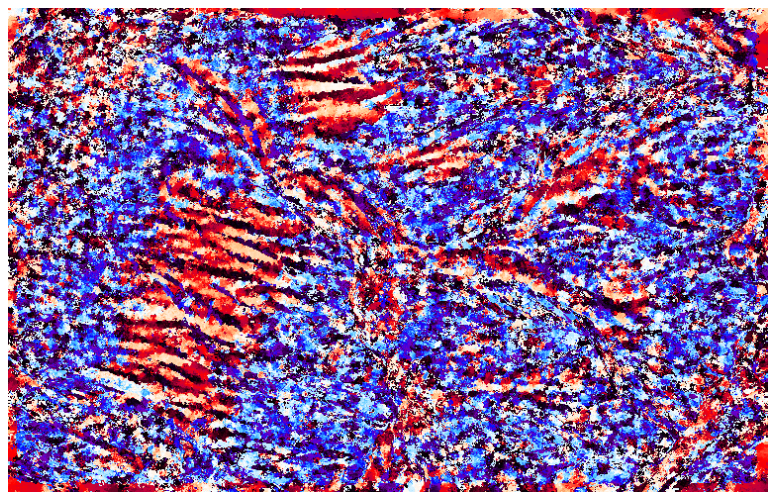

c)

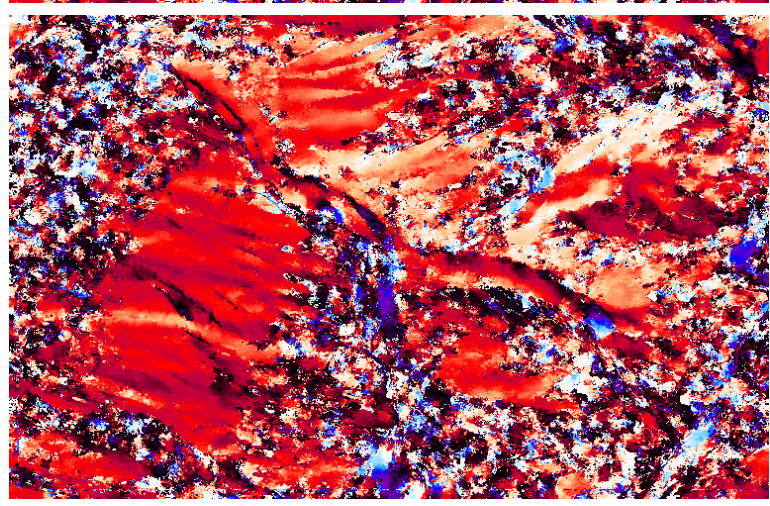

Fig. 2. Orientation map of the local fringe pattern with ERS: a) lowresolution with the local fringe orientation Look-Up-Table in the left upper corner, b) high-resolution, c) sum between the low-resolution and high-resolution components.

provided by the DTM was radar coded and converted into topographic fringes modulo a tunable altitude of ambiguity. A controlled noise level was also introduced on the resulting high-resolution phase and amplitude images.

Figs. 3-a,-b present respectively the real ERS amplitude ( $\sim 20$-meter resolution) and the simulated TerraSAR-X amplitude ( $\sim 2$-meter resolution). As expected, small relief variations become visible in the HR simulated image and the presence of crevasses is noticed. The simulated topographic interferometric phase is presented in Fig. 3-c.

Most of this information is missed by the LR frequency estimation illustrated in Fig. 4-a. It can be observed in the HR estimates (Fig. 4-b) and in the final result (Fig. 4-c).

Fig. 3-d shows the ADAN filtered interferogram obtained by taking into account the estimated deterministic fringe pattern presented in Fig. 4-c. The result is in accordance with the local topography variations observed in the original radar coded altitude information. 
a)

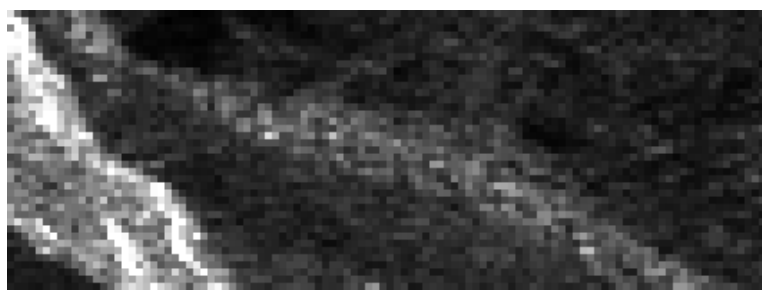

b)

c)

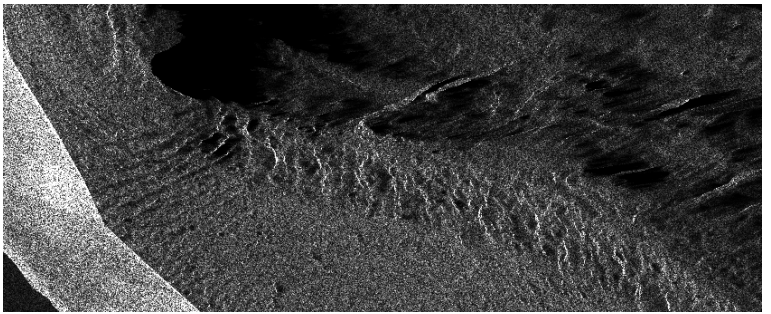

d)
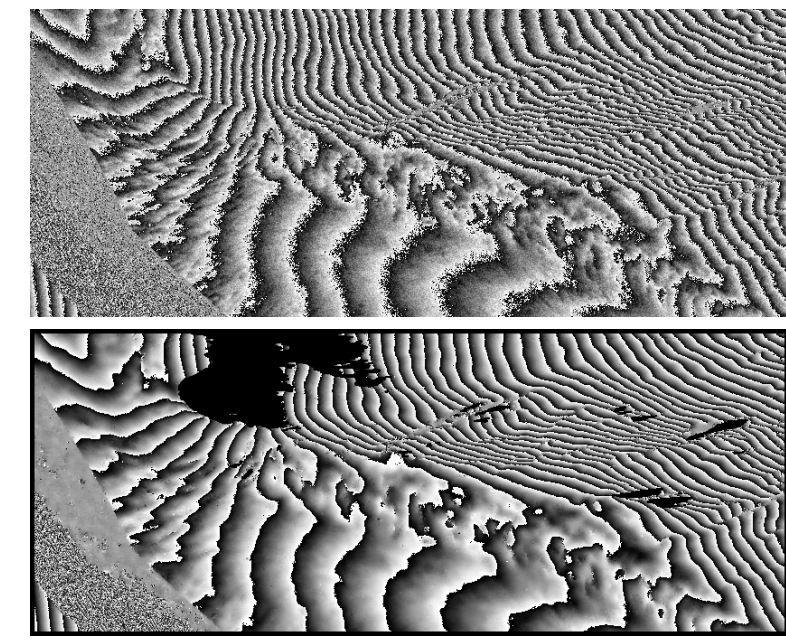

Fig. 3. The Mer-de-glace glacier: a) ERS 5-look amplitude, b) simulated 1-look TerraSAR-X amplitude, c) simulated 1-look TerraSAR$X$ phase, d) TerraSAR-X simulated ADAN filtered phase.

a)

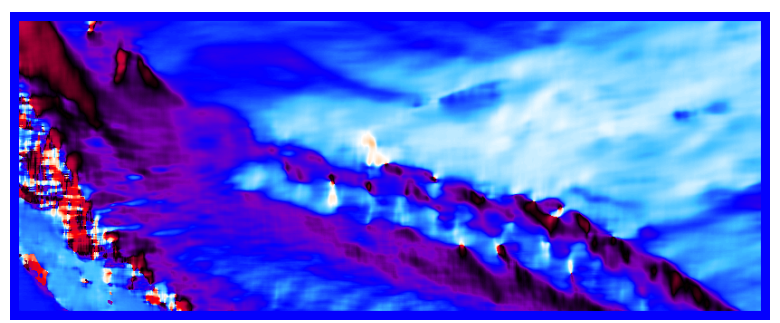

b)

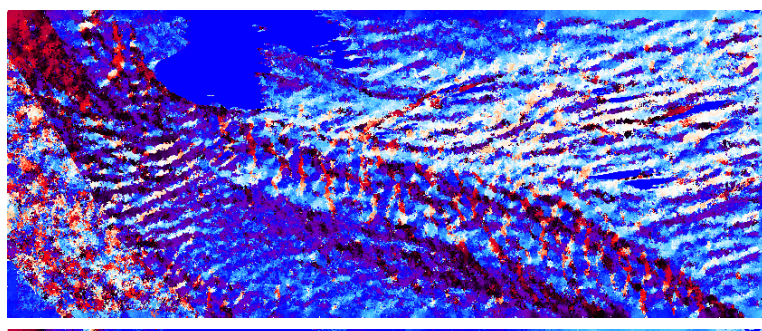

c)

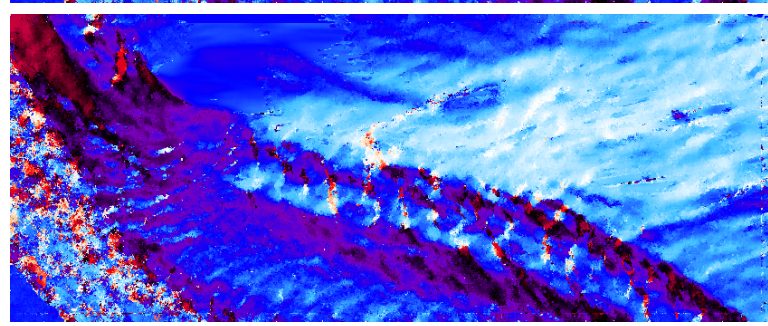

Fig. 4. Orientation map of the local fringe pattern with TerraSAR-X: a) low-resolution, b) high-resolution, c) sum between the low-resolution and high-resolution components.
TABLE II

Processing PARAMETERS IN TERRASAR-X IMAGE SIMULATION.

\begin{tabular}{||c|c||}
\hline \hline \multicolumn{1}{||}{ Parameter } & Value \\
\hline \hline satellite altitude $(\mathrm{km})$ & 514 \\
\hline step on azimuth direction $(\mathrm{m})$ & 2 \\
\hline step on ground range $(\mathrm{m})$ & 2 \\
\hline step on slant range $(\mathrm{m})$ & 1.2 \\
\hline viewing angle at close range (degrees) & 30 \\
\hline altitude of ambiguity (m) & 10 \\
\hline speckle variance in amplitude image & 1 \\
\hline uniform phase noise distribution & $\pm \pi / 4$ \\
\hline
\end{tabular}

\section{CONClusions AND PERSPECTIVES}

The increased performances of future high resolution SAR systems (TerraSAR-X, COSMO-SKYMED or Radarsat-2) together with the developments of appropriate processing algorithms reinforce the potential of SAR interferometry for temperate glacier periodic monitoring. A new method has been proposed to estimate the local frequencies within a HR interferogram. This method allows to measure the local topographic variations which are useful to benefit from the new High Resolution SAR images. In the context of temperate glacier activity monitoring, it can be applied to improve the processing of two kinds of interferograms: small altitude of ambiguity interferograms to derive very accurate DTM and to compute differences, to obtain for instance volume balances. In this case, HR frequency estimation combined with amplitude driven neighborhood techniques provides very good results since most of the sources of phase nonstationarity are revealed by the SAR amplitude which is mostly influenced by the local slope. It will also be useful for high altitude of ambiguity differential interferograms to obtain HR velocity field measurements which require efficient phase filtering and unwrapping methods. In this case, one difficulty that has to be addressed in future works is the presence of phase non-stationarity which are independent from the amplitude information.

\section{ACKNOWLEDGMENT}

This work was supported by the French national project ACIMEGATOR. The authors wish to thank to the European Space Agency for providing ERS SAR data through the Category 1 proposal No.3525.

\section{REFERENCES}

[1] A. Fischer, H. Rott, and H. Björnsson. Observation of recent surges of Vatnajkull, Iceland, by means of ERS SAR interferometry. Annals of Glaciology, 37:69-76, 2003.

[2] E. Trouvé, G. Vasile, M. Gay, P. Grussenmeyer, J.M. Nicolas, T. Landes, M. Koehl, J. Chanussot, and A. Julea. Combining optical and SAR data to monitor temperate glaciers. In IGARSS '05, Seoul, Korea, volume 4, pages 2637-2640, 2005.

[3] G. Vasile, E. Trouvé, M. Ciuc, and V. Buzuloiu. General adaptive neighborhood technique for improving SAR interferometric coherence and phase estimation. J. Opt. Soc. Am. A, 21(8):1455-1464, 2004.

[4] E. Trouvé, J. M. Nicolas, and H. Maître. Improving phase unwrapping techniques by the use of local frequency estimates. IEEE Trans. Geosc. and Rem. Sens., 36(6):1963-1972, 1998.

[5] E. Trouvé, M. Caramma, and H. Maître. Fringe detection in noisy complex interferograms. Applied Optics, 35(20):3799-3806, 1996.

[6] R. Touzi. A review of speckle filtering in the context of estimation theory. IEEE Transactions on Geoscience and Remote Sensing, 40(11):2392-2404, november 2002.

[7] A. Julea, G. Vasile, I. Petillot, E. Trouvé, J.M. Nicolas, M. Gay, and $\mathrm{Ph}$. Bolon. Simulation of SAR images and radar coding of georeferenced information for temperate glacier monitoring. In Optimization of Electrical and Electronic Equipments, OPTIM '06, Brasov, Romania, (accepted), may 2006. 